\title{
The New Applications of Trans-Perineal Ultrasound in Pediatrics
}

\author{
Seyed Ali Alamdaran*
}

Department of Surgical Oncology, Mashhad University of Medical Sciences, Iran

Submission: January 25, 2017; Published: January 31, 2018

*Corresponding author: Seyed Ali Alamdaran, Surgical Oncology Research Center, Mashhad University of Medical Sciences, Mashhad, Iran, Email: AlamdaranA@mums.ac.ir

\section{Mini Review}

The Improvement of spatial resolution of new ultrasound machines were enabled us for better understanding of perineal anatomy, better characterization of perineal lesions and more usage of ultrasound in suspicious perineal abnormalities.

\section{Views and Normal Anatomy}

The child was placed in lithotomy position and trans-perineal ultrasound was done at two sagittal and coronal standard views. In sagittal view, tip of coccyx, anal tract, vagina, urethra and pubis are seen together. In this view, cartilages coccyx is seen as a tapered hypoechoic structure in alignment of sacral bone.
It formed as a curved shape from five body of vertebra. The anal tract has a curved pathway with 45 degree direction to posterior. It has two internal echogenic layers that were surrounded by a thick hypoechoic musculature layer. Vagina has a similar echo pattern with a straight forward direction with minor angulations of perpendicular path; in comparison with anus, internal echogenic mucosa is more prominent and muscular layer is thinner. The female urethra is seen as a small extremely hypoechoic outer layer with small echogenic inner layer at anterior wall of vagina (Figure 1b). On male sex, posterior and anterior urethra is larger and easily is identified as it has specific anatomy in the posterior wall of pubic symphysis (Figure 1).

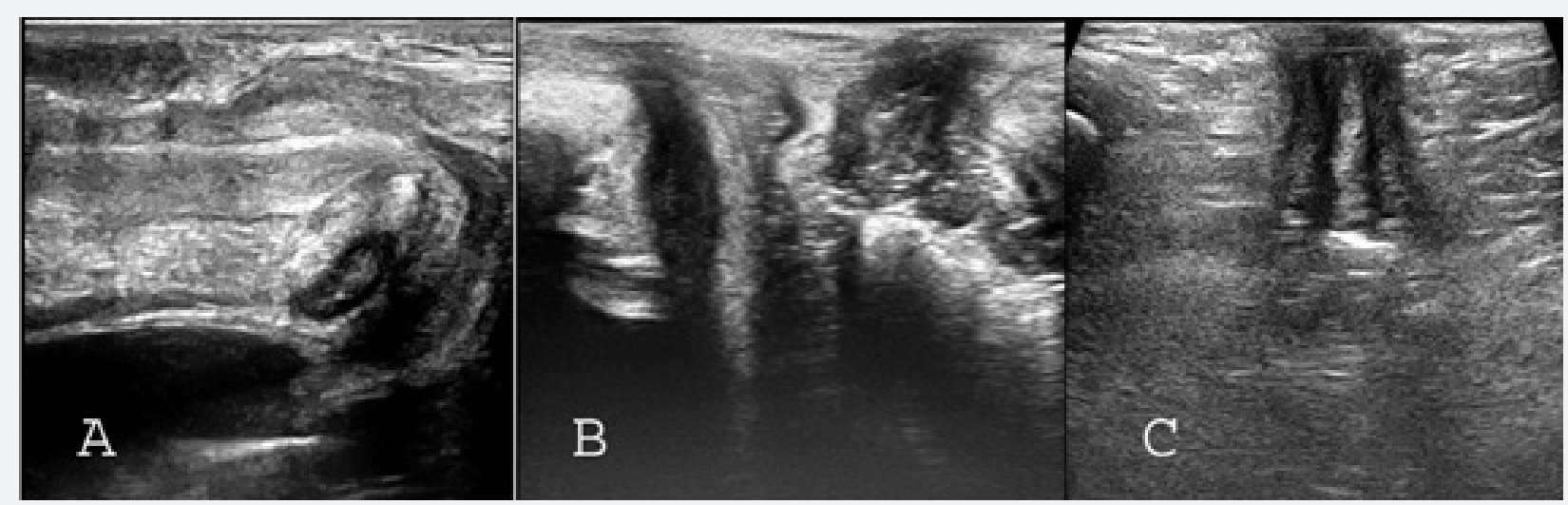

Figure 1: Sagittal and coronal view of ultrasound images of normal perineal area; A-B) sagittal view of male urethra and of female perineum anal tract, vagina, urethra and pubis are seen together C) Coronal view of anal muscle complex and ischiopubic fossa.

On coronal view, you can better evaluate anal musculature and ischiopubic fossa (Figure 1c). The other views are used occasionally. For example; in order to ischial bone examination, the probe must be a parasagittal position with 30 degree angulations of midline.

\section{Applications}

Imperforate anus: In last, the ultrasonography is mainly used In pediatric patients with Imperforate Anus to existence of recto-urethral fistula and determination of the level of disorder (low and high) in according to the distance between perineal skin surface and rectal pouch. Although, there wasn't an exact cut off for their differentiation and there is some diagnostic overlap [1-3]. Todays, the position and state of the anal pit, sphincter complex, internal fistula and type of imperforate anus is easily identified by ultrasound examination (Figure 2). The closed anal pit was detected as a surface multi-layered structure that was exactly similar to gut signature. It is only visualized in the coronal plane as a peripheral hypoechoic layer with two central parallel echogenic lines. 


\section{Juniper Online Journal of Case Studies}

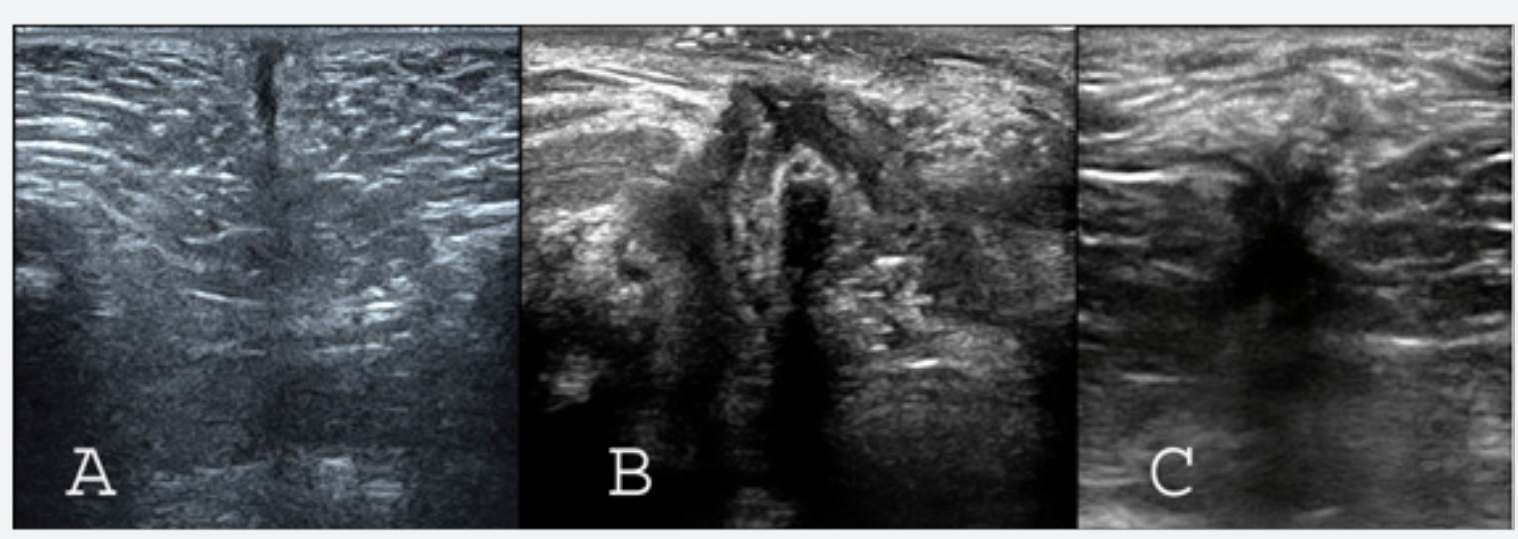

Figure 2: Coronal ultrasound view of Imperforate Anus; A) superfacial anal pit. B) The low type that echogenic wrinkled mucus and gas in the center of hypoechoic muscular sphincter $\mathrm{C}$ ) the high type: deep small contracted muscle sphincter.

The anal sphincter complex was also visible in the coronal plane as a circular muscular tissue in the depth of the subcutaneous perineal area. The muscle complex had 1.8-3.6mm thickness, occasionally asymmetric. Internal fistulas can be identified by changing the rout of the echogenic linear tract of the rectum toward urethra or vagina. The alignment of the middle of the anal pit with the center of the sphincter muscle complex is eccentric or concentric. In the high type of imperforate anus, the rectal pouch is lay above the sphincter muscle, while in the low type of it; the rectal end enters into the sphincter muscle complex and passes partially or completely through it.

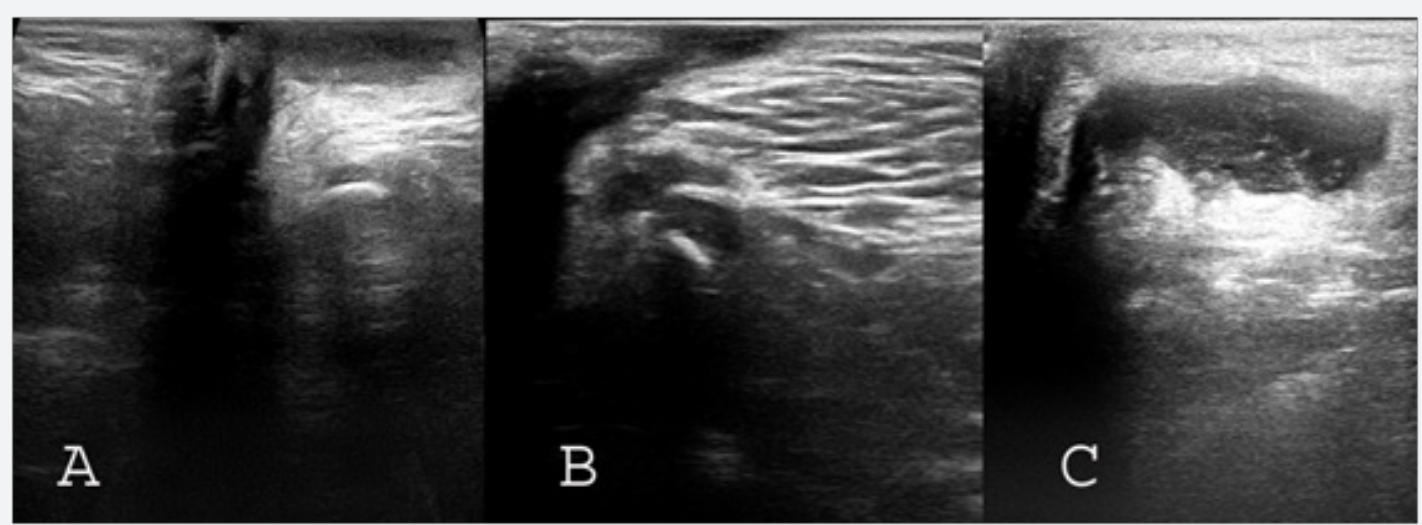

Figure 3: Coronal view of three kind of perineal fistula: A) superfacial submocusal fistula. B) trans-sphincteric fistula C) extrasphincteric type of perianal fistula.

Peri-anal fistula or abscess: Perianal fistula is defined as a tract which may have external and or internal openings. It is usally associated with perianal abscess as local collection related to the anal canal. Submocusal, intersphincteric, transsphincteric, suprasphincteric and extrasphincteric are several kinds of fistula [4]. Transperineal ultrasound can show location of fistula tract and associated abscess (Figure 3). In immune-compromised patients, peri-anal inflammation and anusitis is seen as increasing of thickness of anal wall without localized collection.

Umbigus genitalia: The ultrasound can further delineate anatomy and assess abnormalities that affect the distal genitourinary structures. Detection of presence or absence of vagina can help to differentiation of Disorders of Sex Development and even cloacal anomalies [5].
Vaginal abnormality: Imperforate hymen, vaginal mass, Vaginal Atresia, foreign body and viginitis (Figure 4a) are easily seen with ultrasound.

Urethral lesions: Urethral polyps are seen as an echogenic mass in adjacent to mucosal line. If simultaneous voiding ultrasonography is done, urethral stricture and valve or polyp may show from ventral surface of urethra $[5,6]$.

Caudal regression: The prevalence of various degree of sacrococcyseal hypoplasia in children with bladder dysfunction and constipation was significantly higher compared with normal control group. Normally, cartilages coccyx is so long as that formed a curved hypoechoic structure from five body of vertebra. In coccyx hypoplasia, it is short and straight. Diagnosis of agenesis of coccyx and sacrum is comfortable [7]. 
Presacral mass: Presacral teratoma is seen as a spectral of completely solid until completely cystic mass that laying on around of coccyx and in posterior aspect of anorectal area (Figure 4b).
Ischial bone mass: Tumoral and traumatic lesions of this part of pelvic girdle are relatively common. By transperineal ultrasound can easily evaluate ischial bone and its lesion (Figure 4c).
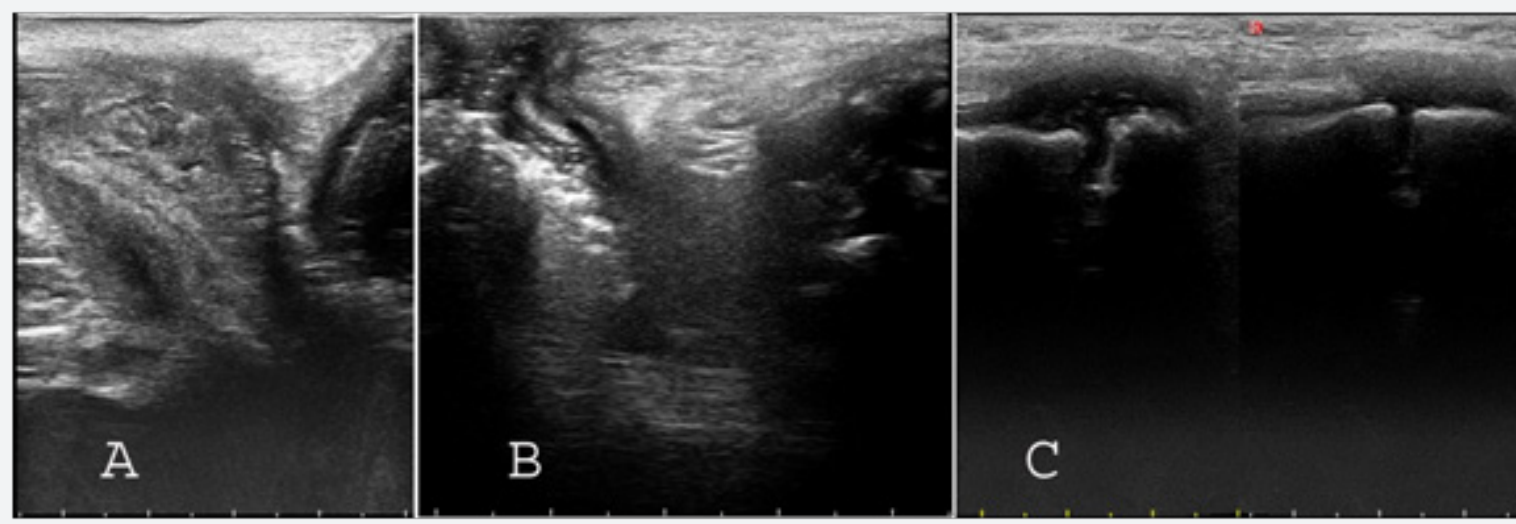

Figure 4: Sagittal view of three various perineal diseases: A) vaginal wall thickninig in obese diabetic child with clinical sign of vaginitis B) posterior perineal mass due to presacral teratoma C) Ischiopubic synchondrosis asymmetry in child with perineal trauma.

Ultrasound guided procedures: Ultrasound-guided wire localization of anal tract in order to less invasive pull-through anorectoplasty in Imperforate anus patients [8] and sono-guided biopsy of pelvic mass; especially presacral teratoma are the most common of interventions in this area in pediatric field.

\section{References}

1. Nemati M, Ataei OR, Aslanabadi S (2013) Assessment of diagnostic value of transperineal ultrasonography in determining type of imperforate anus. J Am Sci 9(10s): 91-95.

2. Haber HP, Seitz G, Warmann SW, Fuchs J (2007) Transperineal sonography for determination of the type of imperforate anus AJR Am J Roentgenol 189(6): 1525-1529.

3. Choi YH, Kim IO, Cheon JE, Kim WS, Yeon KM (2009) Imperforate anus: determination of type using transperineal ultrasonography. Korean ] Radiol 10(4): 355-360.
4. Hwang JY, Yoon HK, Kim WK, Cho YA, Lee JS, et al. (2014) Transperineal ultrasonography for evaluation of the perianal fistula and abscess in pediatric Crohn disease: preliminary study. Ultrasonography 33(3): 184-190.

5. Son JK, Taylor GA (2014) Transperineal ultrasonography. Pediatr Radiol 44:193-201.

6. Teele RL, Share JC (1997) Transperineal sonography in children. AJR Am J Roentgenol 168(5): 1263-1267.

7. M Joodi, Fathi M, Sabzevari A, Nazarzadeh R, Hassani MH, et al. (2017) Intraoperative sonographic guided pull-through anorectoplasty: a novel procedure for imperforate anus and rectourethral fistula: a clinical trial study. Int J Pediatr 5(12): 6195-6200.

8. Alamdaran A, Pourhoseini MT, Jafari SA, Shojaeian R, Feyzi A, et al. (2017) Utrasonographic Evaluation of the Sacrococcyx and Spinal Canal in Children with Constipation. Int J Pediatr 5(12): 6267-6274.

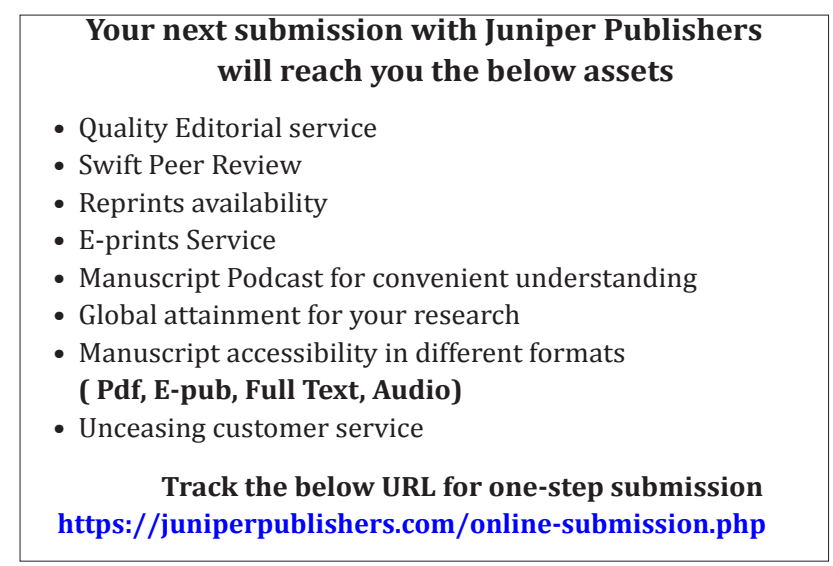

